\title{
ESTRATÉGIAS PSICOEMOCIONAIS COMO MECANISMOS NO ENFRENTAMENTO DO CÂNCER DO COLO UTERINO: REVISÃO DA LITERATURA
}

\section{ARTIGO DE REVISÃO}

MOREIRA, Elisângela Claudia de Medeiros ${ }^{1}$

DENDASCK, Carla Viana ${ }^{2}$

AGUIAR, Cleoci Portela de ${ }^{3}$

ALENCAR, Sandra Maria Ferreira de ${ }^{4}$

PINHEIRO, Dirce Nascimento ${ }^{5}$

OLIVEIRA, Euzébio de 6

\footnotetext{
${ }^{1}$ Mestre em Teoria e Pesquisa do Comportamento. Docente da Universidade do Estado do Pará - UEPA. Doutoranda em Doenças Tropicais na Universidade Federal do Pará - NMT/UFPA.

2 Teóloga. Doutora em Psicanálise Clínica. Pesquisadora do Centro de Pesquisa e Estudos Avançados, São Paulo, SP.

3 Especialista em Doenças Tropicais. Doutoranda em Doenças Tropicais na Universidade Federal do Pará - NMT/UFPA.

${ }^{4}$ Mestre em Gestão Pública. Doutoranda em Doenças Tropicais na Universidade Federal do Pará - NMT/UFPA.

${ }^{5}$ Doutora em Doenças Tropicais. Docente e Pesquisadora na Universidade Federal do Pará - ICS/UFPA.

${ }^{6}$ Doutor em Medicina/Doenças Tropicais. Docente e Pesquisador na Universidade Federal do Pará - UFPA. Pesquisador Colaborador do Núcleo de Medicina Tropical NMT/UFPA.
} 
PINHEIRO, Maria da Conceição Nascimento ${ }^{7}$

MOREIRA, Elisângela Claudia de Medeiros. Et al. Estratégias psicoemocionais como mecanismos no enfrentamento do Câncer do Colo Uterino: revisão da literatura. Revista Científica Multidisciplinar Núcleo do Conhecimento. Ano 04, Ed. 12, Vol. 06, pp. 05-20. Dezembro de 2019. ISSN: 2448-0959, Link de acesso: https://www.nucleodoconhecimento.com.br/saude/estrategiaspsicoemocionais

\section{RESUMO}

O Câncer do Colo Uterino (CCU) é o quarto tipo de câncer de maior incidência entre as mulheres. Esta incidência impulsiona os estudos que possam contribuir tanto para seu diagnóstico e tratamento, quanto para o estudo e/ou o desenvolvimento de estratégias pessoais e sociais, para o enfrentamento dessa doença. Desta forma, este artigo tem como objetivo fazer uma revisão da literatura referente às estratégias psicoemocionais utilizadas pelas mulheres, com diagnóstico de CCU, como mecanismos no enfrentamento da doença. Observa-se que desde o diagnóstico do CCU, não apenas a paciente, mas também seus familiares começam a desenvolver um ciclo estressor, que também requer estratégias psicoemocionais, para o seu enfrentamento, em especial porque essas estratégias, se positivas e bem conduzidas, contribuirão substancialmente para a sustentação do tratamento adequado, obtendo com isso maiores chances de cura. Sendo assim, no enfrentamento, devem ser trabalhados os aspectos cognitivos, o controle emocional, o pensamento positivo e em especial o desligamento do fator estressor. Com base nos achados encontrados, é possível concluir que a maioria das publicações revisadas destaca que neste caso, a importância dos aspectos cognitivos que devem ser trabalhados nessas pacientes, com vista ao fortalecimento, tais aspectos seriam aqueles que fortalecem as

\footnotetext{
${ }^{7}$ Doutora em Neurociências e Biologia Celular. Docente e Pesquisadora do Núcleo de Medicina Tropical - NMT/UFPA.
} 
estratégias psicoemocionais de enfrentamento com ações corretas e positivas, objetivando o sucesso do tratamento, o bem-estar das pacientes e a cura do CCU.

Palavras-Chaves: Câncer do Colo Uterino, CCU, estratégias psicoemocionais, enfrentamento do Câncer.

\section{INTRODUÇÃO}

Estima-se que anualmente 530 mil casos de Câncer de Colo Uterino (CCU) ocorram no mundo, sendo ainda responsável por cerca de 265 mil óbitos por ano, destacandose como a quarta causa de morte por câncer com maior incidência (INCA, 2019). As incidências são mais acentuadas em países em desenvolvimento, em especial na parcela de população com menor nível de escolaridade e de poder econômico (PANOBIANCO et al., 2012). No Brasil a incidência média é de 17,11 casos para cada 100 mil habitantes. Somente em 2018 foram identificados cerca de 16.370 novos casos de CCU no país (INCA, 2019).

O CCU é caracterizado pela replicação desordenada das células que compõe o epitélio de revestimento do colo uterino, comprometendo o tecido subjacente (estroma) e podendo invadir estruturas e órgãos contíguos ou à distância (metástase). Há duas principais categorias de carcinomas invasores do colo do útero, dependendo da origem do epitélio comprometido: o carcinoma epidermoide, tipo mais incidente e que acomete o epitélio escamoso (representa cerca de $90 \%$ dos casos), e o adenocarcinoma, tipo mais raro e que acomete o epitélio glandular (cerca de 10\% dos casos) (WHO, 2014; EGAWA et al., 2015; INCA, 2019).

No Brasil, o controle do CCU constitui uma das prioridades da agenda de saúde do Ministério da Saúde (MS) do país e integra o Plano de Ações Estratégicas para o Enfrentamento das Doenças Crônicas Não Transmissíveis do Ministério da Saúde (DCNT/MS). O MS (2016), por meio da publicação das "Diretrizes para o Rastreamento do Câncer do Colo do Útero", recomenda a realização do exame citopatológico em mulheres assintomáticas, com idade entre 25 e 64 anos, a cada três anos, após dois exames anuais consecutivos com resultados normais. Em caso de 
resultado positivo para lesão de baixo grau, a indicação é de repetição do exame em seis meses. Desde 2014, está disponível, na rede pública, a vacina tetravalente contra os subtipos 6, 11, 16 e 18 do HPV para meninas de 9 a 13 anos; e, a partir de 2017, também para meninos de 11 a 13 anos. Apesar da sua importância epidemiológica, o CCU possui alto potencial de cura quando diagnosticado em estágios iniciais (PIRES; ROCHA, 2017; INCA, 2018).

Seu diagnóstico clássico é realizado através da Anamnese, Exame físico, Exame especular, Citopatologia oncótica (Papanicolau), Colposcopia e Biópsia. Historicamente, os métodos de detecção computadorizados, do status do CCU surgiram como um grande avanço para o tratamento do $\mathrm{CCU}$, e incluem a tomografia computadorizada (TC), ressonância magnética (RM), linfadenectomia e linfangiograma. A TC e a RM são consideradas na atualidade as ferramentas preferidas para a avaliação clínica do CCU em sua fase invasiva (LI; WU; CHENG, 2016).

Apesar das taxas de mortalidade no Brasil serem consideradas ajustadas aos demais países em desenvolvimento, cerca de 4,70 a cada 100 mil habitantes, ainda são consideradas elevadas quando comparadas aos países desenvolvidos, que apresentam menos de 2 casos a cada 100 mil mulheres (INCA, 2018).

No Brasil, a região Norte é a região que apresenta o maior número de casos e de mortes em decorrência dessa doença, sendo considerado o tipo de câncer mais incidente na região, estimando-se 23,97 casos a cada 100 mil mulheres, e óbito em quase $50 \%$ dos casos, cerca de 11,07/100 mil. Nas regiões Centro-Oeste e Nordeste, o CCU ocupa a segunda posição, com taxas de 20,72/100 mil e 19,49/100 mil, respectivamente, e é o terceiro tipo de câncer mais incidente na região Sudeste (11,3/100 mil) e o quarto na região Sul do país (15,17/100 mil) (INCA, 2018).

Por ser um câncer que se desenvolve em decorrência da infecção pelo Papiloma Vírus Humano (HPV) e sua evolução ocorre de forma lenta e assintomática, seu diagnóstico não costuma ocorrer na sua fase inicial, exceto nos casos em que o controle pela mulher, por meio do acompanhamento médico e a realização de exames preventivos 
são realizados de forma periódica (OLIVEIRA et al., 2016). Estima-se que no Brasil, $70 \%$ dos casos com diagnóstico positivo para o CCU, sejam realizados em sua fase avançada (PANOBIANCO et al., 2012). Isso faz com que esse diagnóstico tardio traga expectativas negativas para as mulheres acometidas, quanto ao enfrentamento e o tratamento da doença.

De acordo com a literatura, os aspectos psicoemocionais mais demonstrados pelas pacientes e, inclusive por seus familiares estão relacionados não apenas ao medo da morte, mas também de toda a percepção de que o processo de enfrentamento e do tratamento da doença será doloroso e lhes ocasionarão sensações e debilidades que impactarão o dia a dia de toda a família, gerando, em muitos casos, grandes desordens e instabilidades para todos (MATTOS et al., 2014; SATSANGI; BRUGNOLI, 2017).

O tratamento para cada caso diagnosticado positivamente, deve ser avaliado e orientado por um médico e pela equipe multiprofissional de saúde, especialistas nesse tipo de câncer. Entre os tratamentos mais comuns para o CCU, depois da doença já instalada e geralmente em estado avançado, estão a cirurgia, a radioterapia e a quimioterapia. O tipo de tratamento da doença dependerá de vários fatores, dentre eles destacam-se o estadiamento da doença - que determina o avanço do câncer de uma pessoa, o tamanho do tumor e fatores pessoais, como a idade, o desejo de ter filhos e o quadro clínico geral da paciente (ACS, 2016; INCA, 2018).

Dentro deste contexto, até mesmo para uma melhor compreensão do estado geral destas pacientes, inclusive saber quais são as estratégias psicoemocionais de enfrentamento da doença, que elas estão utilizando, em muitos casos a equipe que está prestando atendimento, ou mais especificamente o psicólogo (a) que está dando assistência à estas mulheres, sempre que possível, aplicam alguns testes psicológicos, para coletar maiores informações psicoemocionais, e que possam ser úteis para o tratamento da doença (RAMOS; PERES, 2013).

Como é possível perceber, o CCU não afeta somente o corpo físico da mulher, compromete também, o estado psicoemocional, que na maioria das vezes, não é 
avaliado efetivamente, impactando negativamente na evolução, e, sobretudo no tratamento, no controle da doença e até mesmo numa possível cura da mesma. Neste contexto, o objetivo deste estudo é investigar a partir da literatura pertinente, quais as estratégias psicoemocionais que são utilizadas pelas pacientes no enfrentamento do diagnóstico e tratamento do CCU.

Diante o exposto as perguntas norteadoras da pesquisa incluem:

1 - Quais as estratégias psicoemocionais de enfrentamento utilizadas pelas mulheres diagnosticadas com CCU?

2 - Quais os testes psicológicos utilizados para identificar as estratégias psicoemocionais utilizadas pelas mulheres diagnosticadas com CCU?

\section{MATERIAL E MÉTODOS}

Este estudo de revisão foi realizado através de levantamentos da literatura mundial referentes às estratégias psicoemocionais de enfrentamento que são utilizadas por mulheres com diagnóstico positivo para o $\mathrm{CCU}$, bem como dos testes psicológicos que são realizados, visando uma melhor compreensão das estratégias psicoemocionais de enfrentamento, que são utilizadas por estas pacientes, para uma melhor abordagem ao tratamento e possível cura desta doença. Foram utilizados para as buscas da literatura os uni termos: Enfrentamento do câncer. Estratégias psicoemocionais $x$ câncer. Estratégias de enfrentamento x câncer. Coping. Câncer de colo uterino. Testagem psicológica. Testes psicológicos em pacientes com CCU. (Todos em português e inglês). Foram selecionados artigos científicos nas bases de busca: Scholar; Google Academic; PubMed; Scielo e em plataformas de informações governamentais que trouxessem maiores informações e dados estatísticos sobre os índices do CCU, bem como sobre os testes psicológicos que são realizados referentes ao enfrentamento da doença. Dentre essas plataformas estão o Instituto Nacional do Câncer (INCA) o Ministério da Saúde (MS), e a Organização Mundial da Saúde (OMS). 
Como critérios de inclusão considerou-se: artigos originais, prioritariamente relacionados ao câncer de colo uterino; artigos recentes publicados exclusivamente nos últimos 10 anos. Foram excluídos ao máximo possível os artigos relacionados com outros tipos de cânceres na mulher e todos os artigos relacionados aos cânceres no homem.

\section{RESULTADOS E DISCUSSÃO}

No total foram encontrados e analisados 180 artigos que abordavam o tema em questão, porém de forma mais genérica. Destes foram selecionados 38 artigos, dentre eles, artigos clínicos e epidemiológicos, publicados em periódicos científicos nacionais e internacionais nos últimos 10 anos que em sua maioria abordavam especificamente: as estratégias psicoemocionais de enfrentamento do $\mathrm{CCU}$, utilizadas pelas mulheres acometidas pela doença e a utilização de testagem psicológica que geralmente são utilizadas para uma melhor compreensão do quadro clínico e psíquico dessas pacientes.

Cabe destacar que os pouquíssimos artigos que foram utilizados, mesmo não tratando das estratégias psicoemocionais de enfrentamento especificamente do CCU, deve-se ao fato de que essas estratégias de enfrentamento, de modo geral são quase sempre as mesmas para outros tipos de câncer também, e estas informações contidas nesses artigos são de extrema relevância. Os artigos selecionados foram lidos na sua íntegra e utilizados neste estudo, de forma sistêmica para compor os tópicos construídos para esta discussão.

\subsection{AS ESTRATÉGIAS PSICOEMOCIONAIS DE ENFRENTAMENTO DO CÂNCER DE COLO UTERINO (CCU)}

O processo estressante, desde a suspeita diagnóstica de um câncer até a reabilitação e o reajuste psíquico da paciente, requer esforços de enfrentamento psicoemocionais por parte da mesma, para cada situação. O câncer, de modo geral, como sendo uma doença crônico-degenerativa pode impor aos pacientes e familiares um grande desafio na adaptação à nova situação, pois o prognóstico da doença e a terapêutica 
a ser escolhida representam uma ameaça à saúde e à integridade do corpo, inclusive podendo levá-lo, em muitos casos, à morte (ALVES et al., 2012; LEITE et al., 2017).

A aplicação correta de técnicas cirúrgicas, radioterápicas, quimioterápicas e biológicas para o tratamento, resultam na cura de mais de $50 \%$ das pacientes diagnosticados com câncer (LONGO, 2011).

Todo contexto da doença propriamente dita e do tratamento podem gerar estresse, trazendo sinais e sintomas como: apatia, depressão, desânimo, sensação de desalento, hipersensibilidade emotiva, raiva, ansiedade, irritabilidade, dentre outros. Neste período é evidenciado que distúrbios psiquiátricos de diferentes ordens podem ser desencadeados, dentre eles: depressão, ansiedade, transtornos obsessivos, etc.. A literatura aponta que de $33 \%$ a $52 \%$ das pacientes apresentam quadros depressivos (KLÜGEL et al., 2017; GHANEM et al., 2019). Esse percentual pode chegar a 65,6\% nos casos de câncer cervical (YANG et al., 2014). A baixa escolarização tende a aumentar os quadros de distúrbios psicológicos (OSANN et al., 2014), bem como as dificuldades para se discutir ou tratar o câncer uterino (PADELA et al., 2014).

Diante disso, o que pode fazer a diferença no resultado de adaptação da mulher ao fato de ser uma paciente oncológica é a forma de enfrentamento psicológico da mesma em relação à doença. Realizar estratégias psicoemocionais de enfrentamento significa que o indivíduo acometido pela doença está tentando superar o que lhe está causando estresse, desconforto, etc. (CHAVES et al., 2009).

O enfrentamento da doença é definido como um processo através do qual o indivíduo administra as demandas da relação pessoa-ambiente, que são avaliadas como estressantes e as emoções que elas geram. $O$ enfrentamento pode ser classificado de acordo com sua função em duas importantes divisões: enfrentamento centrado na emoção e o enfrentamento centrado no problema, embora muitas vezes ocorram simultaneamente, podendo ser mutuamente facilitadores no processo de tratamento e cura do CCU (CHAVES et al., 2009; BINKA et al., 2018). 
A estratégia de enfrentamento focalizada na emoção é definida como um esforço para regular o estado emocional que está associado ao estresse provocado pelo CCU. Esses esforços são dirigidos a um nível somático e/ou a um nível de sentimentos, tendo por objetivo alterar o estado emocional do indivíduo. A principal função dessa estratégia é reduzir a sensação física desagradável provocada por um estado de estresse agudo decorrente da doença (TUNCAY, 2014; KATOWA-MUKWATO et al., 2015; BARNACK-TAVLARIS et al., 2016).

A estratégia de enfrentamento focalizada no problema constitui-se num esforço para atuar na situação que originou o estresse. A função dessa estratégia é alterar o problema existente na relação entre a pessoa e o ambiente que está causando a tensão. A utilização de estratégias de enfrentamento focalizadas no problema ou na emoção depende da avaliação cognitiva da pessoa e da sua capacidade de lidar com a situação estressante de forma mais emocional ou racional possível (COSTA; LEITE, 2009). Desta feita, o comportamento social, a reabilitação e reajuste psíquico/emocional para o enfrentamento psicológico de forma positiva, ou seja, a resiliência pode promover uma melhor adaptação e flexibilidade da paciente, inclusive em relação ao processo de adoecimento frente $\mathrm{CCU}$, com maior responsabilidade e melhor adesão ao tratamento, que podem contribuir significativamente para a qualidade de vida, influenciando diretamente em melhores resultados no combate à doença (AMARO, 2013).

É possível perceber que, para enfrentar essa situação de adoecimento, serão geradas estratégias e mudanças como esforço para aliviar os agentes estressores e que variam de acordo com o grau de desenvolvimento intelectual de cada indivíduo, em relação ao apoio ou não da família e de acordo com suas experiências vivenciadas anteriormente (COSTA; LEITE, 2009).

Diante disso, o que pode fazer a diferença no resultado da adaptação ou não do indivíduo à nova e difícil realidade é a forma de enfrentamento da situação utilizada pelo mesmo. E esta maneira de enfrentar o câncer está relacionada a uma variedade de respostas frente às situações estressantes que estão sendo vivenciadas; as quais são caracterizadas com base na vivência do indivíduo e em reações emocionais 
presentes; o indivíduo se comporta de forma a controlar ou reduzir os efeitos físicos, sociais e emocionais consequentes de tal situação (NUNES, 2010). E estar em "disposição para o enfrentamento aumentado" é o melhor resultado que pode ser encontrado em relação ao enfrentamento e percepção do real sentido de estar com o $\mathrm{CCU}$, significa que a paciente está tentando superar o que lhe está causando estresse (PANOBIANCO et al., 2012).

Dias e Pais-Ribeiro (2019), fizeram uma revisão integrativa e estudaram os aspectos históricos e conceituais dos modelos de enfrentamento do câncer propostos pelos pesquisadores Folkman e Lazarus no século XX, e destacaram que a primeira geração de pesquisadores entendia que as estratégias de enfrentamento do câncer estavam relacionadas aos mecanismos de defesa do ego, sendo concebida como estável e rígida. A segunda geração, a partir de 1960, destacou os comportamentos de estratégias de enfrentamento e seus determinantes cognitivos e situacionais, sendo visto como um processo transacional mais flexível de defesa entre o indivíduo e o ambiente estressor, e a terceira geração, mais recente, têm-se dedicado para o estudo das convergências entre o enfrentamento e a sua personalidade, pois fatores situacionais não são capazes de explicar toda a variação nas estratégias de enfrentamento utilizadas pelas pessoas, isso está diretamente ligado também a personalidade da paciente acometidas por câncer.

$\mathrm{Na}$ década de 80 alguns pesquisadores propuseram um modelo de estratégias psicoemocionais de enfrentamento do câncer que envolve quatro aspectos principais: 1) o enfrentamento como um processo entre indivíduo e ambiente; 2) sua funcionalidade é administrar ao invés de controlar a situação adversa; 3) seus processos implicam em como a situação é percebida e interpretada pelo sujeito acometido; 4) é o processo de enfrentamento se compõe de uma mobilização conjunta de esforços cognitivos e comportamentais para administrar (minimizar, reduzir ou tolerar) os sentimentos que surgem no indivíduo, a partir da sua interação com o ambiente estressor. E foi nesse modelo misto, que acabaram concluindo que os fatores psicológicos têm grande influência nas estratégias de enfrentamento da 
doença, e estão diretamente associados ao sucesso do tratamento, inclusive da cura (YANG et al., 2014).

Nos estudos sobre estratégias de enfrentamento do câncer, observa-se na literatura internacional, a expressão coping referindo-se ao lidar com uma situação ou problema. A tradução para o português que mais se aproxima com o significado do termo original é estratégia de enfrentamento; porém, este pode dar a impressão de que apenas as ações dirigidas à resolução de problemas são consideradas, o que não é o caso. Nesta pesquisa, o termo estratégia de enfrentamento é usado de acordo com o entendimento que é atribuído ao coping, ou seja, considerando todas as formas de se lidar com problemas, sendo adequadas ou não (NUNES, 2010).

As estratégias de enfrentamento também são processos dinâmicos e fundamentais para o sujeito lidar com variadas doenças, dentre elas o $\mathrm{CCU}$, que é a doença foco da presente revisão. O CCU, é um câncer que causa uma desordem fisiopatológica que provoca grandes impactos físicos, psicológicos e sociais na paciente acometida, afetando também toda a família da mesma, que acaba se envolvendo, e que cabe destacar inclusive, que esse apoio é fundamental para o tratamento da mesma. $O$ câncer é uma doença com muitos estigmas para a pessoa que passa por essa experiência e inconvenientes dos mais variados tipos, desde as alterações físicas até as emocionais, que alteram o nível de estresse, e que podem ainda retroalimentar-se. O câncer está inserido dentro de um contexto entendido pela sociedade como "estigma de sofrimento e morte". Para lidar com o estresse provocado pela doença, há diferentes formas de ajustes ao ambiente estressor. O sucesso dessa forma de lidar vai depender das estratégias de enfrentamento (coping) (LEITE et al., 2017).

A estratégia de enfrentamento será efetiva quando o comportamento utilizado pelo sujeito amenizar os sentimentos desconfortáveis, associados a ameaças ou perdas para o mesmo. Por outro lado, será não efetiva se a situação ameaçadora não for manejada de forma eficaz, resultando em crise e, se não for resolvida, podem ocorrer desequilíbrios psicológicos e fisiológicos que podem agravar o quadro clínico emocional do paciente, complicando o andamento do tratamento, e por vezes, até mesmo contribuindo para a morte do indivíduo (SANTOS et al., 2016). 
Adaptar-se ou não a um dado acontecimento, enfrentar situações semelhantes de formas bastante diversificadas dependem de inúmeros fatores que englobam aspectos ambientais, culturais, emocionais, vivências anteriores e com destaque as características pessoais de cada um (COSTA; LEITE, 2009). Roh et al. (2018) apontaram que mulheres que utilizam oração como uma ferramenta de enfrentamento tendem a ter melhor qualidade de vida e resultados no tratamento, indicando que as práticas religiosas e espirituais podem ser um importante fator de proteção contra a tensão da experiência do câncer.

Diante disso, práticas terapêuticas devem ser estruturadas com o intuito de minimizar o impacto causado pela doença e pelo tratamento, sendo então direcionados para auxiliar na adaptação do indivíduo à nova realidade e ao uso favorável ou efetivo das estratégias de enfrentamento (SOUZA, 2009; ALEGRANCE; SOUZA; MAZZEI, 2010). Estes conhecimentos auxiliam no entendimento dos aspectos psicológicos envoltos na doença, e a partir disso, a atuação da equipe multidisciplinar dos profissionais de saúde favorece a transição do paciente e de sua família pelas adversidades decorrentes da enfermidade, auxiliando na melhora do equilíbrio e fortalecimento emocional, do relacionamento interpessoal (profissionais de saúde que prestam o atendimento, outras pessoas com a doença ou não, amigos, etc.) e, consequentemente, leva a um tratamento mais efetivo e consequentemente com resultados mais favoráveis (SOUZA, 2009; BARATTO et al., 2011).

Entre algumas das funções dos profissionais de saúde no processo de cuidado, está o de identificar as estratégias de enfrentamento e sua consequente implicação na evolução clínica do indivíduo visto que são de fundamental importância para o planejamento da assistência psicoemocional em saúde (LEITE et al., 2017). A atuação do psicólogo nesse processo é essencial para avaliar detalhadamente e com olhar crítico os dados obtidos nos testes, a fim de averiguar se forneceram informações seguras e consideráveis para se tomar decisões nos vários contextos. Sendo assim, a testagem psicológica antecede e sustenta a prática, além do conhecimento e do domínio das teorias psicológicas que fundamentam a construção dos testes usados, 
conhecimentos técnicos relacionados aos princípios básicos da psicometria, que têm importância relevada na utilização, compreensão e avaliação dos testes (CFP, 2018).

Dessa forma, é possível concluir que uma análise de todas as variáveis envolvidas no contexto da doença, desde o diagnóstico até o momento final do tratamento, buscando entender quais as estratégias de enfrentamento disponíveis utilizadas pelas pacientes durante o processo, considerando sempre os aspectos pessoais e situacionais que estariam influenciando a emissão de comportamentos de enfrentamento em pacientes com CCU. Desta forma, este conhecimento prévio é imprescindível para que se possa planejar e realizar intervenções psicológicas que contribuam para nortear as atuações dos demais profissionais de saúde e alcançar a melhoria do quadro clínico do paciente de forma completa, em todas as dimensões do ser humano (COSTA; LEITE, 2009).

\subsection{TESTAGEM PSICOLÓGICA EM PACIENTES COM CCU}

O uso de testes psicoemocionais envolve a questão ética e de um saber técnico necessário e fundamentado para a utilização adequada de qualquer tipo de teste. $\mathrm{O}$ profissional tem que ter um conhecimento prévio do contexto em que a testagem se insere, dos aspectos técnicos e das questões psicológicas que envolvem o uso dos testes (ALEGRANCE; SOUZA; MAZEI, 2010).

A avaliação psicológica é compreendida como um amplo processo de investigação, no qual se conhece o avaliado e sua demanda, com o intuito de programar a tomada de decisão mais apropriada do psicólogo. Mais especialmente, a avaliação psicológica refere-se à coleta e interpretação de dados, obtidos por meio de um conjunto de procedimentos confiáveis, entendidos como aqueles reconhecidos pela ciência psicológica, o que nos casos de pacientes com CCU se aplica perfeitamente. Compete ao psicólogo (a), bem preparado e conhecedor do método planejar e realizar o processo avaliativo, com base em aspectos técnicos e teóricos. A escolha do número de sessões necessárias para a sua realização, das questões a serem respondidas, bem como de quais instrumentos/técnicas de avaliação devem ser utilizados (CFP, 2018), e que deve ser baseado nos seguintes elementos: 
1. contexto no qual a avaliação psicológica se insere;

2. propósitos da avaliação psicológica;

3. construtos psicológicos a serem investigados;

4. adequação das características dos instrumentos/técnicas aos indivíduos avaliados;

5. condições técnicas, metodológicas e operacionais do instrumento de avaliação.

Lycke et al. (2017), em estudo sobre preditores de comprometimento cognitivo de pacientes recebendo tratamento curativo para câncer, evidenciam que fatores estressantes do dia ainda podem levar as pacientes com CCU a abandonarem o tratamento, dificultando o acompanhamento e a testagem psicológica, que é de suma importância para essas mulheres para definir fatores preditivos para disfunções cognitivas relacionadas ao câncer.

Pimentel et al. (2011) apontam ainda, como sendo os principais fatores estressantes para essas mulheres acometidas com o CCU: o momento a partir do aparecimento dos primeiros sinais e sintomas da doença; o crescimento do tumor; à medida que 0 diagnóstico foi sendo apresentado e o tratamento se fez necessário; trazendo dificuldades e sofrimento para a paciente. Associado a isso ainda tem os problemas com o chefe, problemas familiares, tais como a violência severa por parte do parceiro, ter que sair de casa, ter uma gravidez não planejada, não receber apoio da família, dentre outros.

Como se sabe não existe um protocolo padrão, já estabelecido de testagem psicoemocional para ser utilizado em pacientes com CCU, para ajudá-las à uma melhor adesão ao tratamento, bem como para estas desenvolverem alternativas saudáveis e positivas de lidar e viver com a doença. No entanto os profissionais, em especial os psicólogos (as) aplicam uma variedade de testes psicológicos, cada um de acordo com a sua conduta que julgar ser a mais adequada. Dentre esses testes podem ser citados o Inventário Fatorial de Personalidade (IFP), o Questionário de Saúde Geral (QSG), o Inventário de Sintomas de Stress de Lipp (ISSL), o Inventário de Percepção de Suporte Familiar (IPSF), o Instrumento de Avaliação de Qualidade 
de Vida (WHOQOL-bref), e a Escala Modos de Enfrentamento de Problemas (EMEP). Cabe novamente destacar que cada um deles tem discutidas e controversas características técnicas e indicações, frente às particularidades de cada população, ou até mesmo de cada paciente especificamente (RAMOS; PERES, 2013).

Por fim, esclarece-se que compete ao psicólogo, após a aplicação dos testes escolhidos analisar criticamente os resultados obtidos, com o intuito de verificar se realmente forneceram elementos seguros e suficientes para a tomada de decisão nos vários contextos de atuação do psicólogo e dos demais membros da equipe multidisciplinar que estão envolvidos no atendimento a paciente com CCU (CFP, 2018).

\section{CONSIDERAÇÕES FINAIS}

Especialmente devido à incidência elevada dos casos de CCU na região Norte e Nordeste do Brasil, estudos que apontem para as formas mais adequadas de enfrentamento desta doença, manifestam-se de extrema importância (OLIVEIRA et al., 2016).

No ínterim desta pesquisa, foi possível observar que as estratégias psicoemocionais realizadas por uma equipe multidisciplinar de saúde, associadas as estratégias psicoemocionais de enfrentamento utilizadas pela própria paciente, auxiliam diretamente no tratamento, e, consecutivamente em seus resultados, que podem ser muito positivos quando todo esse processo for bem conduzido. No entanto, a literatura investigada não apontou um padrão de protocolo ou de estratégias de enfrentamento, estudados e validados, que demonstrem uma melhor eficácia no auxílio ao enfrentamento e tratamento do $\mathrm{CCU}$, que possam ser aplicados para estas pacientes, deixando a cargo dos profissionais que as assistem, escolherem as abordagens terapêuticas a serem consideradas caso a caso, não seguindo assim um padrão mais adequado e/ou até mesmo já preconizado, que inclusive pode ser muito mais eficaz no caso das pacientes com CCU. 
Diante disso, é necessário evidenciar que a adoção de um protocolo padronizado (que deve ter sido muito bem planejado, aplicado/testado, analisado e validado), para o atendimento destes casos de pacientes com CCU, deve ser considerado essencial, já que as decorrências e intercorrências estudadas nesta revisão, tendem a apresentar um mesmo padrão, que por muitas vezes não se demonstra ser eficaz. Desta forma, um protocolo único e padronizado, tenderá a ser mais efetivo para o atendimento da paciente acometida pela doença, seus familiares, bem como para os profissionais da saúde envolvidos no atendimento destes casos.

\section{REFERÊNCIAS}

ALEGRANCE, F. C.; SOUZA, C. B.; MAZZEI, R.L. Qualidade de vida e estratégias de enfrentamento em mulheres com e sem linfodema pós-câncer de mama. Rev. Bras. Cancerol. v. 56, n. 3, p. 341-351, 2010.

ALVES, P. C.; SANTOS, M. C. L.; FERNANDES, A. F. C.. Stress and Coping Strategies for Women Diagnosed with Breast Cancer: a Transversal Study. Online Brazilian Journal of Nursing, [S.I.], v. 11, n. 2, p. 305-18, sep. 2012. ISSN 16764285.

AMARO, L. S. Resiliência em pacientes com câncer de mama: o sentido da vida como mecanismo de proteção. Logos \& Existência, v. 2, n. 2, p. 147-161, 2013.

AMERICAN CANCER SOCIETY (ACS). Last Medical Review: November, 16, 2016 Last Revised: December, 5, 2016.

BARATTO, C. C. et al. (2011). A psicologia no hospital: promoção da qualidade de vida em pacientes oncológicos. Anais da IV Jornada Científica em Psicologia Desafios atuais nas práticas da psicologia (pp.98-109). Santa Cruz do Sul, RS.

BARNACK-TAVLARIS, J. et al. "I have human papillomavirus": an analysis of illness narratives from the Experience Project. Appl Nurs Res. 2016; 30:137-141. 
BINKA, C. et al., - "I always tried to forget about the condition and pretend i was healed": Coping with cervical cancer in rural Ghana. BMC Palliative Care, vol. 17, n. 1, p. 2 - 8. 2018.

BRASIL. Ministério da saúde. Departamento de Vigilância das Doenças Transmissíveis. Nota Informativa №311, de 2016/CGPNI/DEVIT/SVS/MS. Brasília, 2016.

- Instituto Nacional de Câncer (INCA). Estimativas da incidência e mortalidade por câncer 2017-2018. Rio de Janeiro: INCA; 2018.

Ministério da Saúde. Secretaria de Vigilância em Saúde. Departamento de Vigilância de Doenças e Agravos não Transmissíveis e Promoção da Saúde: uma análise da situação de saúde e os desafios para o alcance dos objetivos de desenvolvimento sustentável [recurso eletrônico], 2018.

. Instituto Nacional de Câncer (INCA). Câncer do Colo do Útero. Rio de Janeiro: INCA; 2019.

CHAVES, E. C. et al. Coping: Significados, interferência no processo saúde doença e relevância para a enfermagem. Ver. Esc. Enferm. V.34, n.4, p. 370-375, 2009.

CONSELHO FEDERAL DE PSICOLOGIA (CFP). Resolução 09/2018, que regulamenta a Avaliação Psicológica. 2018.

COSTA, P.; LEITE, R. C. B. O. Estratégias de enfrentamento utilizados pelos pacientes oncológicos submetidos a cirurgias mutiladoras. Revista Brasileira de Cancerologia, v. 55, n. 4, p. 355-364, 2009.

DIAS, E.; N.; PAIS-RIBEIRO, J. O Modelo de Coping de Folkman e Lazarus: Aspectos Históricos e Conceituais. Open Journal Systems. v. 11, n. 2, maio/ago. 2019.

EGAWA, N. et al. Human Papillomaviruses; Epithelial Tropisms, and the Development of Neoplasia. Viruses v. 7, p. 3863-3890, 2015. 
GHANEM, I.; CASTELO, B.; JIMENEZ-FONSECA, P. et al. Coping strategies and depressive symptoms in cancer patients. Clin. Transl. Oncol. (2019). https://doi.org/10.1007/s12094-019-02123-w

KATOWA-MUKWATO, P. et al. Stress and coping with cervical cancer by patients: A qualitative inquiry. International Journal of Psychology and Counselling. Vol. 7(6), p. 94-105, July, 2015. DOI: 10.5897/IJPC2015.0313

KLÜGEL, S.; LÜCKE, C.; META, A. et al. Concomitant psychiatric symptoms and impaired quality of life in women with cervical cancer: a critical review. Int $\mathbf{J}$ Womens Health. 9: 795-805, 2017. doi:10.2147/IJWH.S143368

LEITE, F. M. C. et al. Estratégias de enfrentamento e relação com condições sociodemográficas de mulheres com câncer de mama. Acta paul enferm. v. 25, n. 2, p. 211-217, 2017.

LI, H.; WU, X.; CHENG, X. Advances in diagnosis and treatment of metastatic cervical câncer. J Gynecol Oncol. Jul. 27(4): 27-43, 2016.

LONGO, D. L. Anatomia clínica: Uma abordagem por estudos de casos. MCGRAW HILL EDUCATION.SP: 2011.

LYCKE, M. et al. The distress thermometer predicts subjective, but not objecti ve, cognitive complaints six months after treatment initiation in cancer patients. Journal Of Psychosocial Oncology, New York, v. 35, n. 6, p. 741-757, 2017.

MATTOS, C. T. D. et al. Percepção da mulher frente ao diagnóstico e tratamento do cancer do colo do útero - Subsídios para o cuidado de enfermagem. Revista PróUniverSUS. v.05, n.1, p.27-35, 2014. Jan./Jun.

NUNES, C. M. N. S. O conceito de enfrentamento e a sua relevância na prática da Psiconcologia. Encontro: Revista de Psicologia. 13 (19): 91-102, 2010.

OLIVEIRA, R. C. E. et al. Perfil epidemiológico do HPV da população feminina sexualmente ativa, na faixa etária de 10 a 80 anos. Revista Científica Multidisciplinar 
Núcleo do Conhecimento. Ano 01, Ed. 07, Vol. 04, pp. 21-34, Julho de 2016. ISSN: 2448-0959

OSANN, K.; HSIEH, S.; NELSON, E. et al. Factors associated with poor quality of life among cervical cancer survivors: implications for clinical care and clinical trials. Gynecol Oncol. 135 (2) 266-272, 2014. doi:10.1016/j.ygyno.2014.08.036

PADELA, A. I. et al. Associations between religion-related factors and cervical cancer screening among Muslims in greater chicago. J Low Genit Tract Dis.18(4):326-332, 2014. doi:10.1097/LGT.0000000000000026

PANOBIANCO, M. S. et al. Mulheres com Diagnóstico Avançado do Câncer do Colo do Útero: Enfrentando a Doença e o Tratamento. Revista Brasileira de Cancerologia v.58, n.3, p.517-523, 2012.

PIMENTEL, A. V. A PERCEPÇÃO DA VULNERABILIDADE ENTRE MULHERES COM DIAGNÓSTICO AVANÇADO DO CÂNCER DO COLO DO ÚTERO. Texto Contexto Enferm, Florianópolis, Abr-Jun; 20(2): 255-62, 2011.

PIRES, T. S.; ROCHA, M. S. ASPECTOS ENVOLVIDOS NA VACINAÇÃO CONTRA O HPV. Revista acadêmica Oswaldo Cruz. ano 4, n.14 abril-junho, 2017. ISSN 23578173 (versão on-line).

RAMOS, M.; PERES. G. Métodos Quantitativos e Pesquisa em Ciências Sociais: Lógica e Utilidade do Uso da Quantificação nas Explicações dos Fenômenos Sociais. Mediações, Londrina: 2013.

ROH, S.; BURNETTE, C. E.; LEE, Y. S. Prayer and Faith: Spiritual Coping among American Indian Women Cancer Survivors. Health Soc Work. 43(3):185-192, 2018. doi:10.1093/hsw/hly015.

SANTOS, N. A. R. et al., ESTRATÉGIAS DE ENFRENTAMENTO UTILIZADAS PELOS ENFERMEIROS EM CUIDADOS PALIATIVOS ONCOLÓGICOS: revisão integrativa. Cogitare Enferm. Jul/set; 21(3): 01-08, 2016. 
SATSANGI, A. K.; BRUGNOLI, M. P. Anxiety and psychosomatic symptoms in palliative care: from neuropsychobiological response to stress, to symptoms' management with clinical hypnosis and meditative states. Ann Palliat Med, 7 (1) :75111, 2018. doi: 10.21037/apm.2017.07.01

SOUZA, J. R. (2009). Estudo sobre avaliação de eficácia terapêutica em oncologia: grupo psicoeducacional "Aprendendo a Enfrentar". Dissertação apresentada ao Instituto de Psicologia da Universidade de Brasília, Brasília, DF. 216 p.

TUNCAY, T. Coping and Quality of Life in Turkish Women Living with Ovarian Cancer. Asian Pacific Journal of Cancer Prevention. Vol 15 (9), p. 4005-4012, 2014.

YANG, Y. L. Prevalence and associated positive psychological variables of depression and anxiety among Chinese cervical cancer patients: a cross-sectional study. PLoS One. 9 (4): 94804, 2014. doi: 10.1371/journal.pone.0094804

WHO. Comprehensive Cervical Cancer Control. December, p. 364, 2014.

Enviado: Dezembro, 2019.

Aprovado: Dezembro, 2019. 